UT-11-26

IPMU-11-0129

August, 2011

\title{
Non-anomalous Discrete $R$-symmetry, Extra Matters, and Enhancement of the Lightest SUSY Higgs Mass
}

\author{
Masaki Asano ${ }^{(a)}$, Takeo Moroi ${ }^{(a, b)}$, Ryosuke Sato ${ }^{(a, b)}$ \\ and Tsutomu T. Yanagida ${ }^{(a, b)}$ \\ ${ }^{(a)}$ Department of Physics, University of Tokyo, Tokyo 113-0033, Japan \\ (b) Institute for the Physics and Mathematics of the Universe, \\ University of Tokyo, Kashiwa 277-8568, Japan
}

\begin{abstract}
We consider low-energy supersymmetric model with non-anomalous discrete $R$ symmetry. In such a model, to make the $R$-symmetry non-anomalous, new particles with gauge quantum numbers should be inevitably added to the particle content of the minimal supersymmetric standard model (MSSM). Those new particles may couple to the Higgs boson, resulting in a significant enhancement of the lightest Higgs mass. We show that, in such a model, the lightest Higgs mass can be much larger than the MSSM upper bound; the lightest Higgs mass as large as $140 \mathrm{GeV}$ (or larger) becomes possible.
\end{abstract}




\section{Introduction}

If supersymmetry (SUSY) survives at low-energy scale $(\sim 1 \mathrm{TeV})$, discrete $R$-symmetries $Z_{N R}$ (with $N>2$ ) seem to play an important role. First of all, the constant term in superpotential, that is the gravitino mass, is only controlled by the discrete $R$-symmetries. Therefore, the low-scale breaking of the discrete $R$-symmetries may account for the presence of SUSY at $\mathrm{TeV}$ scale, for example. The discrete $R$-symmetries may play a role of suppressing dangerous dimension 4 operators for proton decays and they also may guarantee the required long lifetime of the lightest SUSY particle as a dark matter candidate\#1 It is known that approximate continuous $R$-symmetries seem an essential component of dynamical SUSY breaking. Those approximate $U(1)_{R}$ symmetries might be realized as an accidental effective symmetry of the discrete $R$-symmetries $Z_{N R}$ with $N>2$.

However, the discrete $Z_{N R}$ symmetries have gauge anomalies in the minimal SUSY standard model (MSSM) [2]. Thus, if it is the case, there is no reason to assume such discrete symmetries as an (almost) exact symmetries. Therefore it seems more interesting to cancel the unwanted anomalies by adding extra matters in the MSSM. In [3] it is shown that the discrete $Z_{4 R}$ symmetry is one of a few candidates. In fact, all gauge anomalies of $Z_{4 R}$ can be canceled out by adding a pair of $\mathbf{5}+\overline{\mathbf{5}}$ chiral multiplets or a pair of $\mathbf{1 0}+\overline{\mathbf{1 0}}$. Their masses are predicted to be at the same order of the Higgsino mass, say $\sim 1 \mathrm{TeV}$. Thus, they must give a significant contribution to low-energy physics. In particular, in the latter solution, up-type Higgs $H_{u}$ can couple to the extra matters in the $\mathbf{1 0}$ multiplet as $W \sim U Q H_{u}$ (where $U$ and $Q$ have the same gauge quantum numbers as right-handed up-type quarks and left-handed quark doublets, respectively).

In this paper, we show that the mass of the lightest Higgs boson can be raised up to $\sim 140 \mathrm{GeV}$ because of the extra Yukawa coupling even when the SUSY-breaking scale is 1 $\mathrm{TeV}$. This will be tested at LHC soon.

\section{Non-Anomalous Discrete $R$-symmetry}

In this section, following [3], we discuss anomaly-free conditions of discrete $R$-symmetry, $Z_{N R}$, in the framework of $S U(5)$ Grand Unified Theories (GUTs). We assume that neutrino masses are explained by seesaw mechanism [4, 5, 6]. Then, the superpotential of the minimal SUSY $S U(5)$ GUT is of the following form:

$$
W_{\mathrm{GUT}} \sim \Phi_{10} \Phi_{10} H+\Phi_{10} \bar{\Phi}_{\overline{\mathbf{5}}} \bar{H}+\bar{\Phi}_{\overline{\mathbf{5}}} \bar{N} \bar{H}+\frac{1}{2} M_{N} \bar{N} \bar{N}+\mu_{H} H \bar{H}
$$

where $M_{N}$ is the Majorana mass of right-handed neutrinos. The quantum numbers of the fields are shown in Table 1 .

\footnotetext{
${ }^{\# 1}$ When the discrete $Z_{N R}$ (with $N>2$ ) is broken down to the $R$-parity $Z_{2 R}$, we may have too many domain walls. For solutions to this domain wall problem, see [1].
} 


\begin{tabular}{|c||c|c|c|c|c|}
\hline & $\Phi_{\mathbf{1 0}}$ & $\bar{\Phi}_{\overline{\mathbf{5}}}$ & $\bar{N}$ & $H$ & $\bar{H}$ \\
\hline$S U(5)_{\mathrm{GUT}}$ & $\mathbf{1 0}$ & $\overline{\mathbf{5}}$ & $\mathbf{1}$ & $\mathbf{5}$ & $\overline{\mathbf{5}}$ \\
\hline$Z_{N R}$ & $\phi_{\mathbf{1 0}}$ & $\bar{\phi}_{\overline{\mathbf{5}}}$ & $\bar{\nu}$ & $h$ & $\bar{h}$ \\
\hline
\end{tabular}

Table 1: The matter content of the supersymmetric $S U(5)$ GUT, and the quantum numbers of the fields. The $Z_{N R}$ charge of the Grassmann coordinate, $\theta$, is denoted as $\alpha$.

To make the model invariant under the discrete $R$-symmetry, the $Z_{N R}$ charges should satisfy the following conditions:

$$
\begin{aligned}
2 \phi_{10}+h & =2 \alpha \bmod N \\
\phi_{10}+\bar{\phi}_{\overline{\mathbf{5}}}+\bar{h} & =2 \alpha \bmod N \\
\bar{\phi}_{\overline{\mathbf{5}}+}+\bar{\nu}+h & =2 \alpha \bmod N \\
2 \bar{\nu} & =2 \alpha \bmod N .
\end{aligned}
$$

Here, we assume that the $\mu_{H}$-term is generated by the Giudice-Masiero mechanism [7], and that $Z_{N R}$-symmetry prevents $\mu_{H}$-parameter from being Planck scale. Then, the following conditions are imposed:

$$
h+\bar{h}=0 \quad \bmod N, \quad \text { and } \quad h+\bar{h} \neq 2 \alpha \quad \bmod N,
$$

which reduce to

$$
2 \alpha \neq 0 \quad \bmod N
$$

Next, we consider the conditions for anomaly cancellation [2]. For $Z_{N R}\left[S U(3)_{C}\right]^{2}$ and $Z_{N R}\left[S U(2)_{L}\right]^{2}$ :

$$
\begin{aligned}
Z_{N R}\left[S U(3)_{C}\right]^{2} & : \frac{3}{2}\left\{3\left(\phi_{\mathbf{1 0}}-\alpha\right)+\left(\bar{\phi}_{\overline{\mathbf{5}}}-\alpha\right)\right\}+3 \alpha=\frac{N}{2} k, \\
Z_{N R}\left[S U(2)_{L}\right]^{2}: & \frac{3}{2}\left\{3\left(\phi_{\mathbf{1 0}}-\alpha\right)+\left(\bar{\phi}_{\overline{\mathbf{5}}}-\alpha\right)\right\}+\frac{1}{2}\{(h-\alpha)+(\bar{h}-\alpha)\}+2 \alpha=\frac{N}{2} k^{\prime},
\end{aligned}
$$

where $k$ and $k^{\prime}$ are integers. Using Eqs. (3), (44) and (6), these condition are rewritten as

$$
\begin{aligned}
& Z_{N R}\left[S U(3)_{C}\right]^{2}: \quad 3 \alpha=\frac{N}{2} k, \\
& Z_{N R}\left[S U(2)_{L}\right]^{2}: \quad \alpha=\frac{N}{2} k^{\prime} .
\end{aligned}
$$

Importantly, Eq. (11) contradicts with the condition (7). Thus, an additional contribution from extra matters is needed to realize a non-anomalous discrete $R$-symmetry in the framework of $S U(5)$ GUT. 
Because the extra matters have gauge quantum numbers, they have to be heavy enough to avoid direct search constraints. If the extra matters are vector-like, their masses can be generated by the Giudice-Masiero mechanism. If so, their masses are expected to be around the mass scale of MSSM superparticles (i.e., $\sim 1 \mathrm{TeV}$ ). In order for the Giudice-Masiero mechanism to work, the following condition should be satisfied:\#2

$$
\phi^{\prime}+\bar{\phi}^{\prime}=0 \bmod N,
$$

where $\phi^{\prime}$ and $\bar{\phi}^{\prime}$ are the $Z_{N R}$ charge of the extra matter multiplets $\Phi^{\prime}$ and $\bar{\Phi}^{\prime}$, respectively.

Extra matters should be embedded in complete multiplets of the GUT group $S U(5)_{\text {GUT }}$ in order not to spoil the gauge coupling unification. In addition, requiring the perturbativity of the gauge coupling constants up to the GUT scale, we cannot introduce too many extra matters. If the masses of extra matters are at $\sim 100 \mathrm{GeV}-1 \mathrm{TeV}$, only a limited number of $\mathbf{5}+\overline{\mathbf{5}}$ and/or $\mathbf{1 0}+\overline{\mathbf{1 0}}$ pairs can be introduced; if a larger representation of $S U(5)_{\mathrm{GUT}}$ is added at $\mu \lesssim 1 \mathrm{TeV}$, the gauge couplings become non-perturbative below the GUT scale. Denoting the numbers of $\mathbf{5}+\overline{\mathbf{5}}$ and $\mathbf{1 0}+\overline{\mathbf{1 0}}$ pairs as $n_{\mathbf{5}^{\prime}}$ and $n_{\mathbf{1 0 ^ { \prime }}}$, respectively, perturbativity of the gauge couplings requires

$$
n_{\mathbf{5}^{\prime}}+3 n_{10^{\prime}} \leq 4
$$

In addition, using Eq. (12), the conditions for the anomaly cancellation are

$$
\begin{aligned}
& Z_{N R}\left[S U(3)_{C}\right]^{2}:\left(3-n_{\mathbf{5}^{\prime}}-3 n_{\mathbf{1 0}^{\prime}}\right) \alpha=\frac{N}{2} k \\
& Z_{N R}\left[S U(2)_{L}\right]^{2}:\left(1-n_{\mathbf{5}^{\prime}}-3 n_{\mathbf{1 0}^{\prime}}\right) \alpha=\frac{N}{2} k^{\prime} .
\end{aligned}
$$

The conditions (7), (13), (14) and (15) are simultaneously satisfied only when $\left(n_{\mathbf{5}^{\prime}}, n_{\mathbf{1 0}}\right)=$ $(1,0),(3,0)$, or $(0,1)$. In these cases, $N$ should be 4 or 20 [3]; for $N=4$ and 20, there exist consistent charge assignments. For $N=4$, for example, one may take $\left(\phi_{\mathbf{1 0}}, \bar{\phi}_{\overline{\mathbf{5}}}, \bar{\nu}, h, \bar{h}, \alpha\right)=$ $(1,1,1,0,0,1)$.

Among three possibilities, we are interested in the case of $\left(n_{\mathbf{5}^{\prime}}, n_{\mathbf{1 0}^{\prime}}\right)=(0,1)$ because the newly introduced 10 multiplet may couple to the up-type Higgs boson if its $Z_{N R}$ charge (denoted as $\phi_{\mathbf{1 0}}^{\prime}$ ) is equal to that of $\Phi_{\mathbf{1 0}}$. Such a charge assignment does not conflict with any of the conditions because $\phi_{\mathbf{1 0}}^{\prime}$ is arbitrary as far as Eq. (12) is satisfied. In the following, we concentrate on the case with an extra pair of $\mathbf{1 0}+\overline{\mathbf{1 0}}$ multiplet, and study the mass of the lightest Higgs boson in such a case.

\section{Higgs Mass}

Now we discuss the lightest Higgs mass, paying particular attention to the contributions of loop diagrams with extra matters inside the loop. As discussed in the previous section, some

\footnotetext{
${ }^{\# 2}$ If SUSY invariant masses for them are allowed, their $R$ charges should satisfy $\phi^{\prime}+\bar{\phi}^{\prime}=2 \alpha$. In this case they do not contribute to the anomalies.
} 
of the fields contained in $\Phi_{\mathbf{1 0}}^{\prime}$ may couple to up-type Higgs $H_{u}$ if it has proper $Z_{N R}$ charge \#3 Then, if its Yukawa interaction is large, we expect a sizable correction to the lightest Higgs mass as in the case of the top and stop [9, 10, 11].

To study the Higgs mass with extra matters, we first decompose $\Phi_{\mathbf{1 0}}^{\prime}$ and $\bar{\Phi}_{\overline{\mathbf{0}}}^{\prime}$ as $\Phi_{\mathbf{1 0}}^{\prime}=$ $Q+U+E$ and $\bar{\Phi}_{\overline{\mathbf{1 0}}}^{\prime}=\bar{Q}+\bar{U}+\bar{E}$, where $Q(\mathbf{3}, \mathbf{2}, 1 / 6), U(\overline{\mathbf{3}}, \mathbf{1},-2 / 3), E(\mathbf{1}, \mathbf{1}, 1), \bar{Q}(\overline{\mathbf{3}}, \mathbf{2},-1 / 6)$, $\bar{U}(\mathbf{3}, \mathbf{1}, 2 / 3)$, and $\bar{E}(\mathbf{1}, \mathbf{1},-1)$ are gauge eigenstates of the standard-model gauge group. (The gauge quantum numbers for $S U(3)_{C}, S U(2)_{L}$ and $U(1)_{Y}$ are shown in the parenthesis.) Then, the relevant part of the superpotential is given by $\# 4$

$$
W=y_{t} t_{R}^{c} q_{L} H_{u}+y_{U} U Q H_{u}+M_{U} \bar{U} U+M_{Q} \bar{Q} Q,
$$

and the soft SUSY breaking terms are\#5

$$
\begin{aligned}
\mathcal{L}_{\text {soft }}= & m_{\tilde{q}}^{2}\left|\tilde{q}_{L}\right|^{2}+m_{\tilde{t}}^{2}\left|\tilde{t}_{R}^{c}\right|^{2}+m_{\tilde{Q}}^{2}|\tilde{Q}|^{2}+m_{\tilde{Q}}^{2}|\tilde{\bar{Q}}|^{2}+m_{\tilde{U}}^{2}|\tilde{U}|^{2}+m_{\tilde{U}}^{2}|\tilde{\bar{U}}|^{2} \\
& +\left(y_{t} A_{t} \tilde{t}_{R}^{c} \tilde{q}_{L} H_{u}+y_{U} A_{U} \tilde{U} \tilde{Q} H_{u}+\text { h.c. }\right),
\end{aligned}
$$

where $q_{L}(\mathbf{3}, \mathbf{2}, 1 / 6)$ and $t_{R}^{c}(\overline{\mathbf{3}}, \mathbf{1},-2 / 3)$ are standard-model quarks in the third generation, which contain left- and right-handed top (s)quarks, respectively. In addition, the "tilde" is for superparticles. (So, $\tilde{Q}$ is the scalar component in the superfield $Q$, for example.)

We presume that the $M_{Q^{-}}$and $M_{U^{-}}$parameters are generated by the Giudice-Masiero mechanism, so the masses of extra matters are expected to be as heavy as the MSSM superparticles. Then assuming a little hierarchy between the electroweak scale and the masses of superparticles, which is suggested by the sparticle search experiments, we estimate the Higgs mass by using the effective field theory approach. Then, the relevant theory describing the energy scale above $M_{\mathrm{SUSY}}$ (which is taken to be the "typical" mass of superparticles) is the MSSM with extra matters, while the low-energy effective theory below $M_{\text {SUSY }}$ is the standard model. Two theories should be matched at $\mu=M_{\text {SUSY }}$ (with $\mu$ being the renormalization scale).

In our analysis, we consider the case that only one light Higgs doublet, which we call the standard-model-like Higgs doublet $H_{\mathrm{SM}}$, remains below $M_{\mathrm{SUSY}}$, which is consistent with the assumption that the low-energy effective theory below $M_{\mathrm{SUSY}}$ is the standard model. The potential of $H_{\mathrm{SM}}$ is denoted as

$$
V_{\mathrm{SM}}=m_{H}^{2}\left|H_{\mathrm{SM}}\right|^{2}+\frac{1}{2} \lambda\left|H_{\mathrm{SM}}\right|^{4}
$$

\footnotetext{
\#3 Because the extra matters couple to the Higgs boson, one should care about the oblique corrections (i.e., so-called $S$ - and $T$-parameters) due to these fields. In the present case, the dominant contribution to the masses of these new particles is from the gauge-invariant operators and hence the oblique corrections become suppressed as these extra particles become heavy. The oblique corrections become small enough if the new particles are as heavy as $\sim 1 \mathrm{TeV}$; for more detail, see $[8]$.

${ }^{\# 4}$ For simplicity, we neglect the effects of possible CP violating phases in the new interaction terms; parameters $y_{U}, M_{Q}, M_{U}$, and $A_{U}$ are all taken to be real.

${ }^{\# 5}$ For simplicity, we assume that the bi-linear SUSY breaking terms for extra matters are negligible.
} 
For the calculation of the lightest Higgs mass, we need to know the coupling constant $\lambda$ at $M_{\text {SUSY }}$; once $\lambda\left(M_{\text {SUSY }}\right)$ is known, Higgs mass is estimated a, \#6

$$
m_{h}^{2}=\lambda\left(m_{h}\right) v^{2}
$$

where $v \simeq 246 \mathrm{GeV}$ is the vacuum expectation value of the standard-model-like Higgs boson. Notice that $\lambda\left(m_{h}\right)$ is related to $\lambda\left(M_{\mathrm{SUSY}}\right)$ by solving renormalization group equation in the framework of the standard model \#7

In the present case, $\lambda\left(M_{\mathrm{SUSY}}\right)$ is given by

$$
\lambda\left(M_{\mathrm{SUSY}}\right)=\frac{1}{4}\left(g_{2}^{2}+g_{1}^{2}\right) \cos ^{2} 2 \beta+\delta \lambda_{\tilde{t}}+\delta \lambda^{\prime},
$$

where $g_{2}$ and $g_{1}$ are gauge coupling constants for $S U(2)_{L}$ and $U(1)_{Y}$, respectively. In addition, $\delta \lambda_{\tilde{t}}$ is the threshold correction at $M_{\mathrm{SUSY}}$ due to the stop loop diagram, while $\delta \lambda^{\prime}$ is that from diagrams with extra matters inside the loop. Taking $m_{\tilde{q}}=m_{\tilde{t}}$ for simplicity, we obtain [14]

$$
\delta \lambda_{\tilde{t}}=\frac{3 y_{t}^{4} \sin ^{4} \beta}{8 \pi^{2}}\left(\frac{A_{t}^{2}}{m_{\tilde{t}}^{2}}-\frac{A_{t}^{4}}{12 m_{\tilde{t}}^{4}}\right) .
$$

We study the effect of the extra matters using one-loop contribution to the effective potential:

$$
\Delta V=\Delta V^{(\mathrm{B})}+\Delta V^{(\mathrm{F})},
$$

where $\Delta V^{(\mathrm{B})}$ and $\Delta V^{(\mathrm{F})}$ are contributions of bosonic and fermionic loops, respectively. $\Delta V^{(\mathrm{B})}$ is given by

$$
\Delta V^{(\mathrm{B})}=\frac{3}{32 \pi^{2}} \operatorname{Tr}\left[\left(\mathcal{M}_{\mathrm{B}}^{2}+\Delta \mathcal{M}_{\mathrm{B}}^{2}\right)^{2}\left\{\ln \left(\frac{\mathcal{M}_{B}^{2}+\Delta \mathcal{M}_{\mathrm{B}}^{2}}{\mu^{2}}\right)-\frac{3}{2}\right\}\right],
$$

where

$$
\mathcal{M}_{\mathrm{B}}^{2}=\operatorname{diag}\left(M_{Q}^{2}+m_{\tilde{Q}}^{2}, M_{Q}^{2}+m_{\tilde{Q}}^{2}, M_{U}^{2}+m_{\tilde{U}}^{2}, M_{U}^{2}+m_{\tilde{U}}^{2}\right)
$$

and

$$
\Delta \mathcal{M}_{\mathrm{B}}^{2}=\left(\begin{array}{cccc}
y_{U}^{2}\left|H_{u}\right|^{2} & 0 & y_{U} A_{U} H_{u}^{*} & y_{U} M_{U} H_{u}^{*} \\
0 & 0 & y_{U} M_{Q} H_{u}^{*} & 0 \\
y_{U} A_{U} H_{u} & y_{U} M_{Q} H_{u} & y_{U}^{2}\left|H_{u}\right|^{2} & 0 \\
y_{U} M_{U} H_{u} & 0 & 0 & 0
\end{array}\right)
$$

\footnotetext{
${ }^{\# 6}$ For the calculation of $\lambda\left(m_{h}\right)$, the most important effect below $M_{\text {SUSY }}$ is from the Yukawa interaction with the top quark. Thus, for our renormalization group analysis, we stop the running of $\lambda$ at $\mu=m_{t}$.

${ }^{\# 7}$ In our numerical analysis, we use the top-quark mass of $m_{t}^{(\text {pole })}=172.9 \mathrm{GeV}$ [12. The pole mass is related to the $\overline{\mathrm{MS}}$ mass as 13 .

$$
\frac{m_{t}^{(\text {pole })}}{m_{t}^{(\overline{\mathrm{MS}})}\left(m_{t}^{(\mathrm{pole})}\right)}=1+\frac{4}{3} \frac{\alpha_{s}\left(m_{t}^{(\text {pole })}\right)}{\pi} .
$$
}


Furthermore,

$$
\Delta V^{(\mathrm{F})}=-\left.\Delta V^{(\mathrm{B})}\right|_{A_{U}=m_{\tilde{Q}}^{2}=m_{\tilde{\bar{Q}}}^{2}=m_{\tilde{U}}^{2}=m_{\tilde{U}}^{2}=0} .
$$

Calculating the coefficient of $\left|H_{u}\right|^{4}$ term in $\Delta V$ and replacing $H_{u} \rightarrow H_{\mathrm{SM}} \sin \beta, \delta \lambda^{\prime}$ is obtained. Because $Q$ and $U$ are in a same multiplet of $S U(5)_{\mathrm{GUT}}$, the relation $M_{Q}=M_{U}$ holds at the GUT scale. This equality is violated by the renormalization group effect below the GUT scale, but the most important effect, i.e., the QCD effect, does not spoil this relation. Thus, we adopt the approximation $M_{Q}=M_{U}$ (at $\mu=M_{\mathrm{SUSY}}$ ). In addition, for simplicity, we approximate $m_{\tilde{Q}}^{2}=m_{\tilde{\tilde{Q}}}^{2}=m_{\tilde{U}}^{2}=m_{\tilde{\tilde{U}}}^{2}$. Then, $\delta \lambda^{\prime}$ is given by

$$
\begin{aligned}
\delta \lambda^{\prime}= & \frac{3 y_{U}^{4} \sin ^{4} \beta}{8 \pi^{2}} \ln \left(\frac{M_{U}^{2}+m_{\tilde{U}}^{2}}{M_{U}^{2}}\right) \\
& -\frac{y_{U}^{4} \sin ^{4} \beta}{32 \pi^{2}} \frac{A_{U}^{4}-\left(8 M_{U}^{2}+12 m_{\tilde{U}}^{2}\right) A_{U}^{2}+8 M_{U}^{2} m_{\tilde{U}}^{2}+10 m_{\tilde{U}}^{4}}{\left(M_{U}^{2}+m_{\tilde{U}}^{2}\right)^{2}} .
\end{aligned}
$$

One can see that, with a relevant choice of parameters, $\delta \lambda^{\prime}$ becomes positive and an enhancement of the lightest Higgs mass happens.

To see how large the Higgs mass can be, we calculate $m_{h}$. To make our discussion simple, we take

$$
m_{\tilde{t}}^{2}=m_{\tilde{q}}^{2}=m_{\tilde{Q}}^{2}=m_{\tilde{\tilde{Q}}}^{2}=m_{\tilde{U}}^{2}=m_{\tilde{U}}^{2} \equiv m_{\mathrm{SUSY}}^{2},
$$

and the tri-linear coupling constants are parametrized as

$$
A_{t}=a_{t} m_{\mathrm{SUSY}}, \quad A_{U}=a_{U} m_{\mathrm{SUSY}} .
$$

For our numerical calculation, we take $M_{\mathrm{SUSY}}=m_{\mathrm{SUSY}}$.

In Fig. 1, we plot the lightest Higgs mass on $y_{U}$ (at $\mu=M_{\text {SUSY }}$ ) vs. $a_{U}$ plane for several values of $\tan \beta$, taking $m_{\mathrm{SUSY}}=1 \mathrm{TeV}, M_{U}=M_{Q}=1 \mathrm{TeV}$, and $a_{t}=a_{U}$. If the Yukawa coupling constants are too large, they diverge below the GUT scale. Requiring the perturbativity (i.e., $y_{U}^{2} \lesssim 4 \pi$ ) below the GUT scale, the upper bound on $y_{U}\left(m_{\mathrm{SUSY}}\right)$ is obtained; in the figure, such a bound is also shown. In addition, as one can see from Eq. (21), $\delta \lambda_{\tilde{t}}$ takes its maximal value when $a_{t}=\sqrt{6}$; results for such a case are shown in Fig. 2.

One can see that the radiative correction due to the extra particles may drastically change the lightest Higgs mass; $m_{h}$ can be significantly enhanced compared to the case of the MSSM [15, 16]. We note here that the lightest Higgs mass is sensitive to the $A_{U}$-parameter. In particular, when $a_{U} \sim 3$ and $y_{U} \sim 1$, the lightest Higgs mass becomes as heavy as $\sim 140 \mathrm{GeV}$ even if we assume the perturbativity of the Yukawa coupling constant up to the GUT scale. Such a value of $m_{h}$ is above the MSSM bound on the lightest Higgs mass, which is $\sim 125 \mathrm{GeV}$ if $m_{\mathrm{SUSY}}=1 \mathrm{TeV}$ [17]. We have also checked that a larger value of $m_{h}$ is also possible for a different value of $M_{U}$ or $m_{\tilde{U}}$. As the ratio $m_{\tilde{U}}^{2} / M_{U}^{2}$ becomes large, the logarithmic term in Eq. (27) is enhanced, resulting in a larger value of $m_{h}$. For example, when $M_{U}=500-750 \mathrm{GeV}$ (and $\left.m_{\mathrm{SUSY}}=1 \mathrm{TeV}\right), m_{h}$ can be made as large as $145-150 \mathrm{GeV}$. 


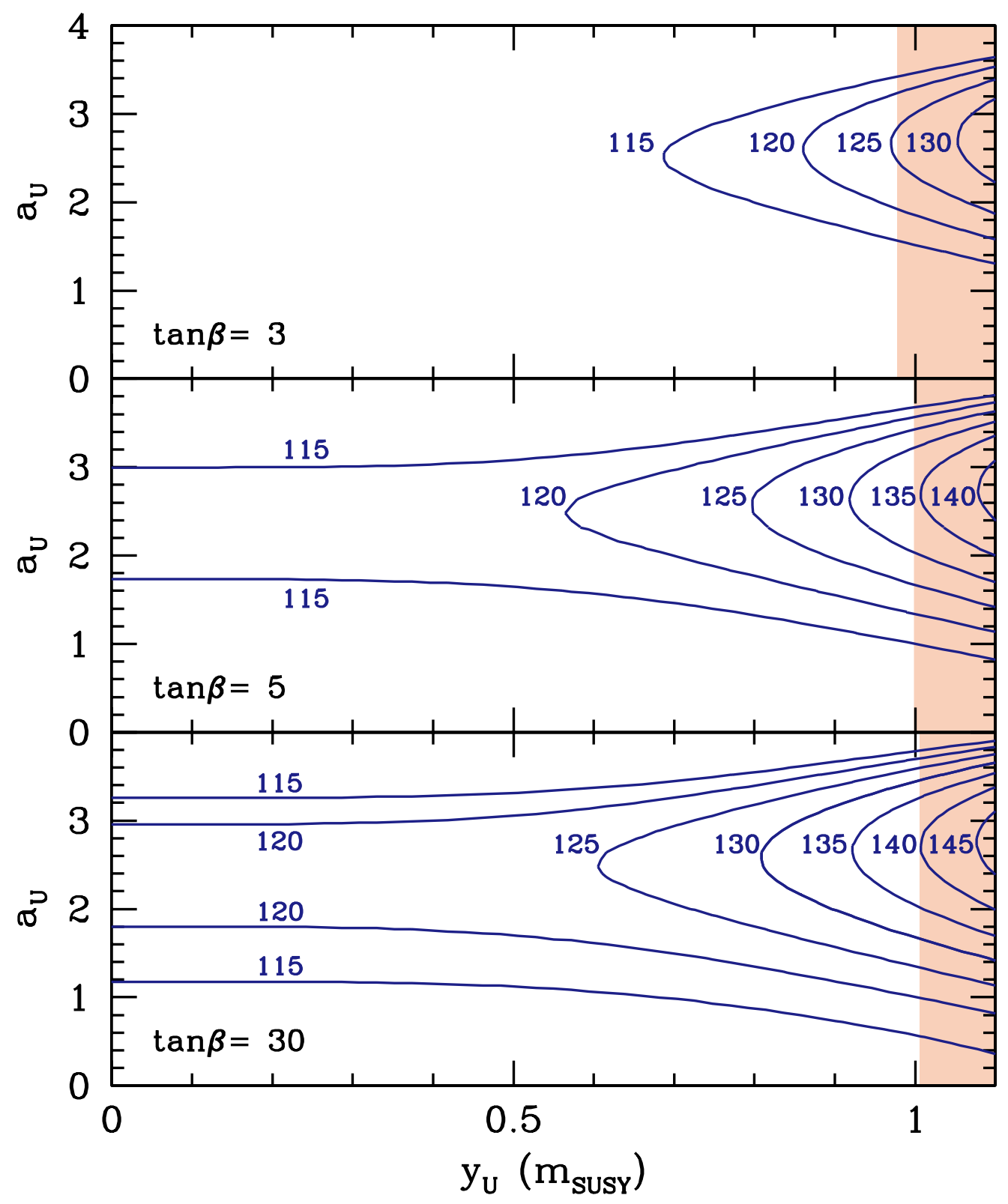

Figure 1: Contours of constant $m_{h}$ on $y_{U}$ vs. $a_{U}$ plane for $\tan \beta=3,5$, and 30 . Here, we have taken $m_{\mathrm{SUSY}}=M_{U}=M_{Q}=1 \mathrm{TeV}$, and $a_{t}=a_{U}$. In the shaded region, $y_{U}$ becomes non-perturbative below the GUT scale. Numbers in the figure are the lightest Higgs mass in units of GeV. 


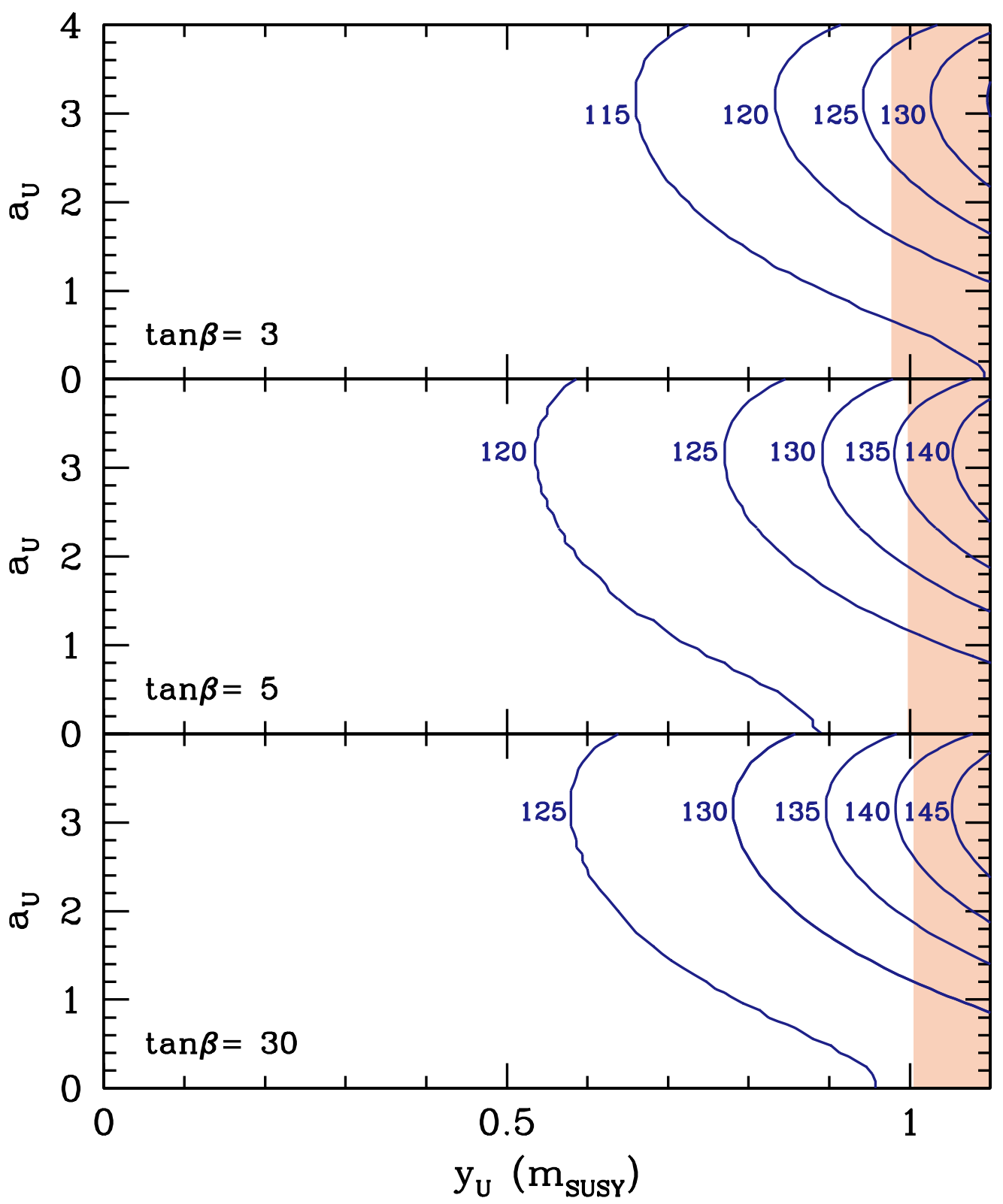

Figure 2: Same as Fig. 1, except for $a_{t}=\sqrt{6}$. 


\section{Discussion}

In this paper, we have discussed the lightest Higgs mass in a model with a non-anomalous discrete $R$-symmetry. For the cancellation of the gauge anomaly, extra particles should be added to the MSSM; we have seen that the gauge anomaly can be cancelled out by adding $\mathbf{1 0}+\overline{\mathbf{1 0}}$ multiplet of $S U(5)_{\text {GUT. }}$. In such a model, the SUSY invariant mass term arises for the $\mathbf{1 0}+\overline{\mathbf{1 0}}$ multiplet via the Giudice-Masiero mechanism, and the particles in the $\mathbf{1 0}+\overline{\mathbf{1 0}}$ multiplet becomes as light as MSSM superparticles. We have paid particular attention to the lightest Higgs mass in such a model, and we have seen that $m_{h}$ can become as large as $\sim 140 \mathrm{GeV}$ (or larger).

This fact has a great impact on the study of SUSY models because the Higgs mass is the crucial check point of low-energy SUSY and also because the LHC experiment is expected to find Higgs boson in near future. We have shown that the significant enhancement of the Higgs mass is possible if extra particles from 10 multiplet of $S U(5)_{\text {GUT }}$ exist; such a modification is well-motivated to realize a non-anomalous discrete $R$-symmetry. In particular, the ATLAS group recently observed $\sim 2.8 \sigma$ excess of the Higgs-like events in the mass range of $\sim 120-140 \mathrm{GeV}$ [18]. If the existence of the Higgs boson heavier than the MSSM bound is confirmed, it is strongly suggested to look for extra particles in $\mathbf{1 0}+\overline{\mathbf{1 0}}$ multiplet.

Our estimation of the Higgs mass is based on the renormalization-group analysis with taking account of the leading-order threshold correction at $\mu=M_{\mathrm{SUSY}}$; the sub-dominant contributions are expected to be suppressed by powers of $v / M_{\mathrm{SUSY}}$ or $v / M_{U}$. Such subdominant contributions may slightly change the lightest Higgs mass. For more precise determination of $m_{h}$, the full one-loop calculation of the effective potential is needed, which is beyond the scope of this paper. However, in order to estimate the accuracy of our results, we have compared our results (for the case without extra matter) with those of FeynHiggs package [19, 20, 21, 22] which is expected perform a precise calculation of the Higgs mass in the framework of the MSSM. We found that the difference between two results are within $\sim 5 \mathrm{GeV}$.

Before closing this paper, several comments are in order. First comment is on the stability of extra particles. If we strictly adopt the superpotential given in Eq. (16) and soft SUSY breaking terms given in Eq. (17), the lightest extra particle becomes stable. If a charged or colored particle becomes stable, it may conflict with cosmological constraints. However, the extra particles can decay into standard-model quarks or leptons (and weak boson) if they

slightly mix with standard-model particles. Because the $Z_{N R}$ charges of the extra particles are same as or opposite to that of the standard-model fermions, such mixing naturally exists.

We have seen that the enhancement of the lightest Higgs mass becomes significant when $\tan \beta$ is large. This fact has an advantage if we take the muon $(g-2)$ anomaly seriously. If we compare the face values of the measured value of the muon anomalous magnetic moment with the theoretical prediction, they have $3.3 \sigma$ discrepancy [23]. In low-energy supersymmetric models, SUSY contribution to the muon anomalous magnetic moment becomes sizable in particular when $\tan \beta$ is large [24, 25], which may be the origin of the muon $(g-2)$ anomaly. In the present set up, thus the muon $(g-2)$ anomaly may be solved with realizing the 
Higgs mass much larger than the MSSM upper bound. This is a big contrast to the case with a singlet Higgs, i.e., the so-called the next to the MSSM (NMSSM), which may also enhance the lightest Higgs mass; in the NMSSM, $\tan \beta$ is required to be relatively small for the enhancement of $m_{h}$ [26].

Acknowledgments: The authors thank J. Evans and M. Ibe for useful discussion. This work is supported by Grant-in-Aid for Scientific research from the Ministry of Education, Science, Sports, and Culture (MEXT), Japan, No. 22244021 (M.A., T.M., and T.T.Y.), No. 22540263 (T.M.), and also by the World Premier International Research Center Initiative (WPI Initiative), MEXT, Japan. The work of R.S. is supported in part by JSPS Research Fellowships for Young Scientists.

\section{References}

[1] M. Dine, F. Takahashi and T. T. Yanagida, JHEP 1007 (2010) 003.

[2] L. E. Ibanez and G. G. Ross, Phys. Lett. B 260 (1991) 291.

[3] K. Kurosawa, N. Maru and T. Yanagida, Phys. Lett. B 512 (2001) 203.

[4] T. Yanagida, in Proc, of the Workshop on the Unified Theory and Baryon Number in the Universe, ed. O. Sawada and A. Sugamoto, (KEK report 79-18, 1979).

[5] M. Gell-Mann, P. Ramond and R. Slansky, in Supergravity, ed P. van Nieuwenhuizen and D.Z. Freedman, (North Holland, Amsterdam, 1979)

[6] P. Minkowski, Phys. Lett. B 67 (1977) 421.

[7] G. F. Giudice and A. Masiero, Phys. Lett. B 206 (1988) 480.

[8] J. Evans, M. Ibe and T.T. Yanagida, in preparation.

[9] Y. Okada, M. Yamaguchi and T. Yanagida, Prog. Theor. Phys. 85 (1991) 1.

[10] H. E. Haber and R. Hempfling, Phys. Rev. Lett. 66 (1991) 1815.

[11] J. R. Ellis, G. Ridolfi and F. Zwirner, Phys. Lett. B 257 (1991) 83.

[12] K. Nakamura et al. [Particle Data Group], J. Phys. G 37 (2010) 075021.

[13] H. Arason, D. J. Castano, B. Keszthelyi, S. Mikaelian, E. J. Piard, P. Ramond and B. D. Wright, Phys. Rev. D 46 (1992) 3945.

[14] Y. Okada, M. Yamaguchi and T. Yanagida, Phys. Lett. B 262 (1991) 54.

[15] T. Moroi and Y. Okada, Mod. Phys. Lett. A 7 (1992) 187. 
[16] T. Moroi and Y. Okada, Phys. Lett. B 295 (1992) 73.

[17] M. S. Carena and H. E. Haber, Prog. Part. Nucl. Phys. 50 (2003) 63.

[18] The ATLAS collaboration, ATLAS-CONF-2011-112 (July, 2011).

[19] S. Heinemeyer, W. Hollik and G. Weiglein, Comput. Phys. Commun. 124 (2000) 76.

[20] S. Heinemeyer, W. Hollik and G. Weiglein, Eur. Phys. J. C 9 (1999) 343.

[21] G. Degrassi, S. Heinemeyer, W. Hollik, P. Slavich and G. Weiglein, Eur. Phys. J. C 28 (2003) 133.

[22] M. Frank, T. Hahn, S. Heinemeyer, W. Hollik, H. Rzehak and G. Weiglein, JHEP 0702 (2007) 047.

[23] K. Hagiwara, R. Liao, A. D. Martin, D. Nomura and T. Teubner, J. Phys. G 38 (2011) 085003.

[24] U. Chattopadhyay and P. Nath, Phys. Rev. D 53 (1996) 1648.

[25] T. Moroi, Phys. Rev. D 53 (1996) 6565 [Erratum-ibid. D 56 (1997) 4424].

[26] See, for example, U. Ellwanger, C. Hugonie and A. M. Teixeira, Phys. Rept. 496 (2010) 1. 